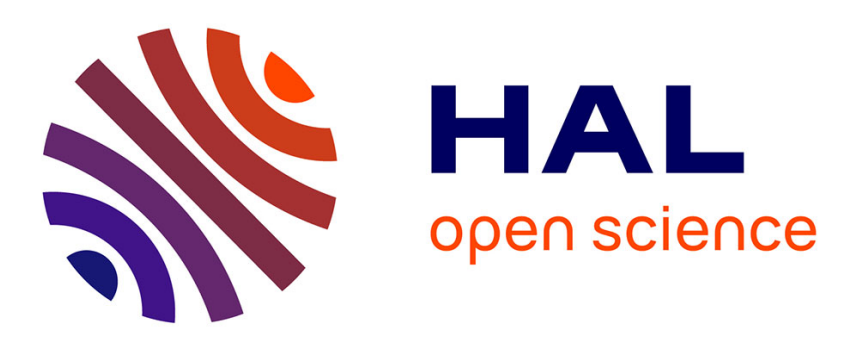

\title{
Boundary control of a Boussinesq equation for a crystal growth problem - aspects of numerical solution
}

\author{
Gunter Baerwolff
}

\section{To cite this version:}

Gunter Baerwolff. Boundary control of a Boussinesq equation for a crystal growth problem - aspects of numerical solution. International Journal of Computer Mathematics, 2008, 85 (03-04), pp.329-343. 10.1080/00207160701203393 . hal-00545348

\section{HAL Id: hal-00545348 \\ https://hal.science/hal-00545348}

Submitted on 10 Dec 2010

HAL is a multi-disciplinary open access archive for the deposit and dissemination of scientific research documents, whether they are published or not. The documents may come from teaching and research institutions in France or abroad, or from public or private research centers.
L'archive ouverte pluridisciplinaire HAL, est destinée au dépôt et à la diffusion de documents scientifiques de niveau recherche, publiés ou non, émanant des établissements d'enseignement et de recherche français ou étrangers, des laboratoires publics ou privés. 


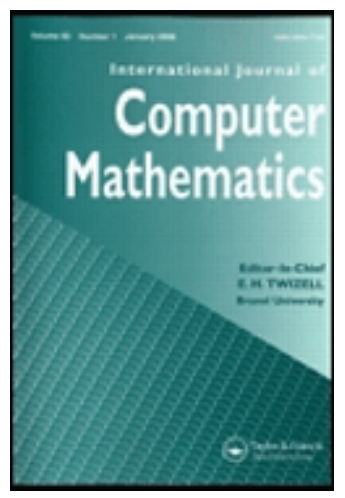

\section{Boundary control of a Boussinesq equation for a crystal growth problem - aspects of numerical solution}

\begin{tabular}{|r|l|}
\hline Journal: & International Journal of Computer Mathematics \\
\hline Manuscript ID: & GCOM-2006-0147.R1 \\
\hline Manuscript Type: & Original Article \\
\hline $\begin{array}{r}\text { Date Submitted by the } \\
\text { Author: }\end{array}$ & 08-Dec-2006 \\
\hline Komplete List of Authors: & Baerwolff, Gunter; TU Berlin, Institute for Mathematics \\
\hline Keywords: & tanja, ulrike, matthias, gabi, guenter \\
\hline $\begin{array}{l}\text { Note: The following files were submitted by the author for peer review, but cannot be converted } \\
\text { to PDF. You must view these files (e.g. movies) online. }\end{array}$ \\
\hline tandf_revision_gb.tex \\
\hline
\end{tabular}

\section{S) ScholaroNE \\ Manuscript Central}


International Journal of Computer Mathematics

Vol. 00, No. 00, Month 200x, 1-12

\title{
Boundary control of a Boussinesq equation for a crystal growth problem - aspects of numerical solution
}

\author{
Günter Bärwolff \\ Institut für Mathematik, Technische Universität Berlin, D-10623 Berlin, Germany \\ (Received 00 Month 200x; revised 00 Month 200x; in final form 00 Month 200x)
}

\begin{abstract}
An optimization problem for a Boussinesq equation system will be formulated. We are looking for a temperature profile or an appropriate velocity on the boundary of the considered region of the thermal coupled flow problem to induce a forced convection which implies a velocity field close to a prescribed one. For such tracking type optimization problems with tracking type minimization functionals the evaluation of the first order necessary optimality condition leads to an optimality system consisting of the forward (primal) and adjoint (dual) mathematical model.

Beside the derivation of the optimality system we discuss aspects of numerical solution, e.g. the spatial and time discretization and the iteration method for the solution of the resulting coupled nonlinear primal and dual problem in this paper.

The optimization concept will be applied to a crystal growth flow and results of two-dimensional and three-dimensional model problems will be presented.
\end{abstract}

Keywords: FV-discretization of the Boussinesq equation system, pressure Poisson equation, free and forced convection, Boussinesq approximation, optimization of partial differential equations, crystal melt flow, FV-discretization of the primal and dual problem, iterative solution method of a KKT-system

AMS Subject Classifications: 65M60, 65M06, 76D05, 76D55, 76K10, 76R05, 76R10

\section{Introduction}

During the growth of crystals crystal defects were observed under some conditions of the growth device. A transition from the two-dimensional flow regime of a crystal melt in axisymmetric zone melting devices to an unsteady three-dimensional behavior of the velocity and temperature field was found experimentally. This behavior leads to striations as undesirable crystal defects. To avoid such crystal defects it is important to know the parameters, which guarantee a stable steady two-dimensional melt flow during the growth process.

A good overview of the crystal growth problems which will be discussed in this paper as an real-wolrd application problem is given in the [6].

There are several possibilities for parameter finding, for example by experiments or systematic parameter studies. All this methods include only a finite number of parameters. In this paper optimization problems will be discussed and the goal is to find optimal functions, i.e. infinite optimal parameters. From the practical crystal production process it is known that an unsteady behavior of the melt and vortices near the fluid-solid-interphase decreases the crystal quality. Thus it makes sense to look for example for (i) flows, which are nearly steady or close to a desired state and (ii) flows, which have only a small vorticity in a certain region of the melt zone.

This leads to tracking type optimization problems (i) with functionals like

$$
J\left(\vec{u}, \theta_{c}\right)=\frac{1}{2} \int_{0}^{T} \int_{\Omega}|\vec{u}-\overline{\vec{u}}|^{2} d \Omega d t+\frac{\alpha}{2} \int_{0}^{T} \int_{\Gamma_{c}}\left(\theta_{c}^{2}+\left[\partial_{t} \theta_{c}\right]^{2}\right) d \Omega d t
$$

Email: baerwolf@math.tu-berlin.de

International Journal of Computer Mathematics ISSN 0020-7160 print/ISSN 1029-0265 online (c) 200x Taylor \& Francis http://www.tandf.co.uk/journals DOI: $10.1080 / 0020716 Y Y x x x x x x x x$ 
and problems with optimization functionals of the form

$$
J\left(\vec{u}, \theta_{c}\right)=\frac{1}{2} \int_{0}^{T} \int_{\Omega_{s}}|\operatorname{curl} \vec{u}|^{2} d \Omega d t+\frac{\alpha}{2} \int_{0}^{T} \int_{\Gamma_{c}}\left(\theta_{c}^{2}+\left[\partial_{t} \theta_{c}\right]^{2}\right) d \Omega d t .
$$

$\vec{u}=(u, v, w)$ is the velocity vector field in the melt and $\overline{\vec{u}}$ is a desired state, which represents a physically favorable flow situation. $\Omega$ is the melt flow region with the boundary $\Gamma$, and $\Omega_{s}$ is a certain part of $\Omega$. $\theta_{c}$ is the control temperature on the control boundary $\Gamma_{c} \subset \Gamma$ and is called the control variable. $\partial_{t} \theta_{c}$ stands for the time derivative of $\theta_{c}$. The first term of $J$ defined by (1) measures the distance between the desired state $\overline{\vec{u}}$ and the state $\vec{u}$ as a result of the mathematical model for the melt flow, and the second term penalizes the need of energy. From the mathematical point of view the second term is a regularization term with the regularization parameter $\alpha$. In the case (2) of $J$ a velocity field with small vorticity is the desired state.

The discussed methods of deriving of optimality systems following [7], [8] and the iterative algorithms of the evaluation of necessary optimality conditions are investigated by the solution of typical crystal growth problems. Because of the difficulties to construct or to prescribe desirable flow fields we use $\overline{\vec{u}}$ which we got by a certain forward solution of the Boussinesq equation system or we set $\overline{\vec{u}}$ equal to zero. But with the optimization strategy we are ready to compute an optimal control for a given desirable flow field $\overline{\vec{u}}$ by crystal growth engineers.

\section{Mathematical model}

The crystal melt is described by the Navier-Stokes equations for an incompressible fluid using the Boussinesq approximation coupled with the convective heat conduction equation and the diffusion equation. Heat conductivity and viscosity might depend on the temperature but this dependence is neglected here. Thus we have a Boussinesq equation system for the velocity $\vec{u}=(u, v, w)$, the pressure $p$ and the temperature $\theta$

$$
\begin{aligned}
& \partial_{t} \vec{u}+(\vec{u} \cdot \nabla) \vec{u}-\Delta \vec{u}+\nabla p-G r \theta \vec{g}=0, \\
& -\operatorname{div} \vec{u}=0, \\
& \partial_{t} \theta+\vec{u} \cdot \nabla \theta-\frac{1}{P r} \Delta \theta-f=0,
\end{aligned}
$$

on the space-time cylinder $\Omega_{T}=\Omega \times(0, T)$. The vector $\vec{g}$ is directed in the $z$-direction, i.e. $\vec{g}=(0,0,1)$. We will now discuss the case $f=0$ ( $f$ stands for an energy source) because we are mainly interested in boundary control. $\vec{u}$ is the velocity vector. $u, v, w$ and $p$ are the primitive variables of the velocity vector and the pressure, $\rho$ and $\theta$ denote the density and the temperature, $G r$ is the Grashof number, $\operatorname{Pr}$ is the Prandtl number. $\vec{u}, p, \theta$ are the state variables.

The boundary conditions are of the form

$$
\begin{aligned}
& u=u_{d}, v=v_{d}, w=w_{d} \quad \text { on } \Gamma_{T}, \\
& \theta=\theta_{c} \quad \text { on the control boundary } \Gamma_{c} \times[0, T], \\
& \theta=\theta_{d}, \quad \text { on } \Gamma_{d} \times[0, T],
\end{aligned}
$$

where $\Gamma_{T}$ means the boundary-time cylinder $\Gamma \times[0, T]$ and $\Gamma=\Gamma_{c} \cup \Gamma_{d}, \Gamma_{c} \cap \Gamma_{d}=\emptyset$.

Dirichlet data on the control boundary as a first choice are used, because there are some results in [10], where was solved an inverse problem to find a heating strategy with a heat flux density $q\left(\theta_{c}\right)$ for catching a given temperature profile $\theta_{c}$ on the boundary. Our idea is to find optimal temperature profiles during the optimization method, described in this paper. And after that the identification of an optimal heat flux 
density $q$ which leads to our determined optimal boundary temperature profile with the method of [10] can be used to find the optimal heating strategy.

In the case of the Czochralski crystal growth technique with $u_{d}, v_{d}, w_{d}$ we have the possibility to describe a certain crystal and crucible rotation. If we prescribe a rotation we have a superposition of natural and forced convection for the Czochralski melt. In contrast to the Czochralski technique $u_{d}, v_{d}, w_{d}$ are equal to zero in the zone melting flow, i.e. we have no slip flow boundary conditions. The quite different geometrical and material parameters distinguish tho Czochalski from the zone melting technique (s. also the tables below).

The initial velocity field $\vec{u}_{0}$ is assumed as the neutral position of the crystal melt $(\vec{v}=\mathbf{0})$ and an initial temperature field $\theta_{0}$, which solves the non convective stationary heat conduction equation with the boundary conditions $\theta=\theta_{d}$ on $\Gamma_{d}$ and $\theta=\theta_{0}$ on $\Gamma_{c}$, is chosen. The material properties and the dimensionless parameters for the investigated crystals close the initial boundary value problem for the description of the melt flow.

\section{Optimization}

For example we construct an optimization system for the case of a boundary temperature profile as a control variable and a cost functional (1). For the calculus of optimization and the derivation of an optimality system we use the above described dimensionless mathematical model (3)-(5) with the boundary conditions (6). For $t=0$ we have the initial condition $\vec{u}=\mathbf{0}$ and a temperature field, which solves the non convective heat conduction equation with the given temperature boundary conditions $\theta=\theta_{0}$ on $\Omega$.

The optimization problem considered in the present work is given by

$$
\text { (P) }\left\{\begin{array}{l}
\min J\left(\vec{u}, \theta_{c}\right) \\
\text { s.t. }(3)-(6) .
\end{array}\right.
$$

It is important to note that the optimization problem (7) is not restricted to the description of the above mentioned problems of crystal growth. With (7) it is possible to treat broadly flow optimization at what natural convection arises.

To derive the first order necessary optimality conditions for this optimization problem we formally utilize the Lagrange technique. The related Lagrangian in the primitive setting is given by

$$
\begin{array}{r}
L\left(\vec{u}, p, \theta, \theta_{c}, \vec{\mu}, \xi, \kappa, \chi\right)=J\left(\vec{u}, \theta_{c}\right)+\left\langle\vec{\mu}, \partial_{t} \vec{u}+(\vec{u} \cdot \nabla) \vec{u}-\Delta \vec{u}+\nabla p-\operatorname{Gr} \theta \vec{g}\right\rangle_{\Omega_{T}} \\
-\langle\xi, \operatorname{div} \vec{u}\rangle_{\Omega_{T}}+\left\langle\kappa, \partial_{t} \theta+\vec{u} \cdot \nabla \theta-\frac{1}{\operatorname{Pr}} \Delta \theta-f\right\rangle_{\Omega_{T}}+\left\langle\chi, \theta-\theta_{c}\right\rangle_{\Gamma_{c T}},
\end{array}
$$

where $\langle\cdot, \cdot\rangle_{\Gamma_{c T}}$ and $\langle\cdot, \cdot\rangle_{\Omega_{T}}$ denote appropriate duality pairings, and $\vec{\mu}, \xi, \kappa$ and $\chi$ are Lagrange parameters. For example for $\vec{u}, p$ and $\theta$ sufficiently regular one has

$$
<\vec{\mu}, \partial_{t} \vec{u}+(\vec{u} \cdot \nabla) \vec{u}-\Delta \vec{u}+\nabla p-G r \theta \vec{g}>_{\Omega_{T}}=\int_{\Omega_{T}}\left[\partial_{t} \vec{u}+(\vec{u} \cdot \nabla) \vec{u}-\Delta \vec{u}+\nabla p-G r \theta \vec{g}\right] \cdot \vec{\mu} d \Omega d t .
$$

A precise functional analytic setting, also containing the convergence analysis of the solution algorithms proposed in the subsequent sections will be given in a forthcoming paper [5], see also $[8,11]$.

THEOREM 3.1 (first order optimality condition) The solution $\left(\vec{u}, p, \theta, \theta_{c}, \vec{\mu}, \xi, \kappa, \chi\right)$ of the primal boundary value problem (3)-(6) and the dual problem

$$
-\partial_{t} \vec{\mu}-\Delta \vec{\mu}+(\nabla \vec{u})^{t} \vec{\mu}-(\vec{u} \cdot \nabla) \vec{\mu}+\nabla \xi+\kappa \nabla \theta=-(\vec{u}-\overline{\vec{u}}) \quad \text { in } \quad \Omega_{T},
$$


with the boundary condition $\vec{\mu}=\mathbf{0}$ on $\Gamma_{T}$, and the final condition $\vec{\mu}(T)=\mathbf{0}$ in $\Omega$ and

$$
\begin{gathered}
-\operatorname{div} \vec{\mu}=0 \quad \text { in } \quad \Omega_{T}, \\
-\partial_{t} \kappa-\frac{1}{P r} \Delta \kappa-\vec{u} \cdot \nabla \kappa=G r \vec{g} \cdot \vec{\mu} \quad \text { in } \quad \Omega_{T},
\end{gathered}
$$

with the boundary condition $\kappa=0$ on $\Gamma_{T}$, and the final condition $\kappa(T)=0$ in $\Omega$, and the choice of $\chi$ as

$$
\chi=-\frac{1}{\operatorname{Pr}} \partial_{\nu} \kappa \quad \text { on } \quad \Gamma_{c} \times[0, T]
$$

with $\partial_{\nu}$ as the derivative in the direction of the outer normal, and

$$
\alpha\left(-\partial_{t t} \theta_{c}+\theta_{c}\right)=\chi\left(=-\frac{1}{P r} \partial_{\nu} \kappa\right) \quad \text { on } \quad \Gamma_{c} \times[0, T]
$$

with the time boundary conditions

$$
\theta_{c}(0)=\theta_{0} \quad \text { and } \quad \partial_{t} \theta_{c}(T)=0
$$

for the control, where $\theta_{0}$ means a temperature distribution on $\Gamma_{c}$ at the beginning of the melting process, which is physical and technological founded,

are critical or stationary points, which fulfill the first order optimality condition

$$
\nabla L\left(\vec{u}, p, \theta, \theta_{c}, \vec{\mu}, \xi, \kappa, \chi\right)=\overrightarrow{0} .
$$

The proof of theorem 3.1 is given in the paper [4].

THEOREM 3.2 (existence and uniqueness)

The problem (3)-(6) with the initial conditions $\vec{u}=\vec{u}_{0}, \theta=\theta_{0}$ admits for a given $\theta_{c}$ a weak solution, if the initial data for the velocity and temperature satisfy the assumptions

$$
\vec{u}_{0} \in H, \quad \theta_{0} \in \mathcal{D},
$$

where $H$ is the closure of

$$
V=\left\{\vec{u} \mid \vec{u} \in\left(C^{\infty}(\Omega)\right)^{n}, \operatorname{div} \vec{u}=0\right\}
$$

in the Hilbert-space $\left(L^{2}(\Omega)\right)^{n}$ and $\mathcal{D}$ is the closure of

$$
W=\left\{\theta \mid \theta \in C^{\infty}(\Omega)\right\}
$$

in $L^{2}(\Omega)$. In the case $n=2$ the solution is unique.

The proof of theorem 3.2 is given in [1] based on results of [2], [3].

Remark 1 We have shown the existence and uniqueness of the coupled primal dual problem under appropriate assumptions on the boundary and initial data and we will publish the results in a forthcoming paper [5]. In [9] was shown the existence and uniqueness of a solution of the adjoint model for a flow problem without a thermal coupling. Minimization functionals of type (1) and (2) are investigated for example in [8] and [13].

Now we can summarize, and the complete optimization system consists of 
1) the forward model with the Boussinesq equations (3),(4),(5), the boundary condition (6) and the given initial state for the velocity field $\vec{u}$, the pressure $p$ and the temperature $\theta$, and

2 ) the adjoint model with the equations $(9),(10),(11),(12)$, and the boundary and final conditions for the adjoint variables $\vec{\mu}, \xi, \kappa$ and the control $\theta_{c}$,

and we will call it the optimality or Karush-Kuhn-Tucker system (KKT system). The main reason for the considered quadratic functionals is the technological aim of the crystal growth methods. This kind of chosen functionals is the key for the investigation of qualitative mathematical questions - for example like existence of a minimum - which are still under consideration.

\section{To the numerical solution method of the full problem}

The primal and dual initial/final boundary value problems are discretized in space by a finite volume method on staggered grids (see [4]).

Next let us describe the numerical solution methods used to solve the primal and dual problems (s. also steps ii)-iv) in the iterative loop below). For the description of the time integration method we denote by $t_{i}:=i \tau, i=0, \ldots Z$ an equidistant time grid on $[0, T]$, where $\tau:=\frac{T}{Z}$ for some $Z \in \mathbb{N}$. Unknown quantities are supplied with superscripts. For the primal problem we apply a semi-implicit time discretization scheme. Convective terms are treated explicitly, conductive terms implicitly. We obtain for $n=0, \ldots, Z-1$

$$
\begin{aligned}
& \frac{\vec{u}^{n+1}}{\tau}-\Delta \vec{u}^{n+1}+\nabla p^{n+1}-G r \theta^{n+1} \vec{g}=\frac{\vec{u}^{n}}{\tau}-\left(\vec{u}^{n} \cdot \nabla\right) \vec{u}^{n}, \\
& -\operatorname{div} \vec{u}^{n+1}=0, \\
& \frac{\theta^{n+1}}{\tau}-\frac{1}{P r} \Delta \theta^{n+1}=\frac{\theta^{n}}{\tau}-\left(\vec{u}^{n} \cdot \nabla\right) \theta^{n} .
\end{aligned}
$$

Taking the divergence in (15) leads to

$$
-\Delta p^{n+1}=\frac{1}{\tau} \operatorname{div} \overrightarrow{\hat{u}}
$$

where

$$
\overrightarrow{\hat{u}}=\vec{u}^{n}+\tau\left[\left(\vec{u}^{n} \cdot \nabla\right) \vec{u}^{n}+G r \theta^{n+1} \vec{g}\right] .
$$

Because of the no slip velocity boundary conditions we have homogenous Neumann boundary conditions for the pressure Poisson equation (18). For a given boundary control $\theta_{c}$ temperature $\theta$ and velocity field $\vec{u}$ at the time level $n$ the computation of the time step " $n \rightarrow n+1$ " means the solution

a) of a Helmholtz equation for $\theta^{n+1}$,

b) of a Poisson equation for $p^{n+1}$ and

c) of three scalar Helmholtz equations for the components of $\vec{u}^{n+1}$.

For the time discretization of the dual system we use the scheme

$$
\begin{aligned}
& \frac{\vec{\mu}^{n-1}-\vec{\mu}^{n}}{\tau}-\Delta \vec{\mu}^{n-1}+\left(\nabla \vec{u}^{n-1}\right)^{t} \vec{\mu}^{n}-\left(\vec{u}^{n-1} \cdot \nabla\right) \vec{\mu}^{n}+\nabla \xi^{n-1}=-\kappa^{n-1} \nabla \theta^{n}-\left(\vec{u}^{n-1}-\overline{\vec{u}}\right) \\
& -\operatorname{div} \vec{\mu}^{n-1}=0, \\
& \frac{\kappa^{n-1}-\kappa^{n}}{\tau}-\vec{u}^{n-1} \cdot \nabla \kappa^{n}-\frac{1}{\operatorname{Pr}} \Delta \kappa^{n-1}=\operatorname{Gr} \vec{g} \cdot \vec{\mu}^{n},
\end{aligned}
$$

for $n=Z, \ldots, 1$, where we have $\vec{\mu}=\mu(T)=0$ and $\kappa=\kappa(T)=0$ for $n=Z$. Starting with given adjoint 
variables $\mu, \kappa$ and the temperature $\theta$ at the time level $n$ and $\vec{u}^{n-1}$ the computation of $\vec{\mu}^{n-1}, \xi^{n-1}$ and $\kappa^{n-1}$ means again four Helmholtz equations and one Poisson equation. The time discretization (15)-(17) is in a certain sense adjoint to the time discretization (20)-(22) of the dual problem (s. also [4]).

With the resulting adjoint temperature $\kappa$ it is possible to determine a boundary control $\theta_{c}$ by evaluating the condition

$$
\hat{J}^{\prime}\left(\theta_{c}\right)=0
$$

for the Gradient

$$
\hat{J}^{\prime}\left(\theta_{c}\right)=\alpha\left(\partial_{t t} \theta_{c}+\theta_{c}\right)-\chi
$$

of the reduced cost functional

$$
\hat{J}\left(\theta_{c}\right)=J\left(\vec{u}\left(\theta_{c}\right), \theta_{c}\right)
$$

In other words we have to solve the equation (12).

For the representation of the used iteration method we denote with $\mathcal{H}:=-\partial_{t t}+i d$ a solution operator, which describes the solution of the two point boundary value problem (12),(13) on $\Gamma_{c T}$, i.e.

$$
\mathcal{H}\left(\theta_{c}\right)=\chi \quad \text { or } \quad \theta_{c}=\mathcal{H}^{-1}(\chi) .
$$

The inversion of $\mathcal{H}$ i.e. the solution of the two point boundary value problem (12),(13) on $\Gamma_{c T}$ is done by a finite volume method in space and time, where the boundary conditions $\theta_{c}(\gamma, 0)=\theta_{c 0}$ and $\partial_{t} \theta_{c}(\gamma, T)=0$ for $\gamma \in \Gamma_{c}$ are taken. Iterative methods of the form

i) choose a suitable start value of $\theta_{c}$,

ii) solve the forward problem and get $[\vec{u}, \theta]\left(\theta_{c}\right)$

iii) solve the adjoint problem and get $[\vec{\mu}, \kappa](\vec{u}, \theta)$

update of $\theta_{c}$ by $\theta_{c}:=\sigma_{r} \theta_{c}+\left(1-\sigma_{r}\right) \mathcal{H}^{-1}(\chi)$, with a damping parameter $\left.\sigma_{r} \in\right] 0,1[$,

iv) until convergence, go to ii),

are used (Picard method). As a stopping criterion for the Picard iteration method we use

$$
\frac{\max _{\Gamma_{c} \times[0, T]}\left|\theta_{c}^{n+1}-\theta_{c}^{n}\right|}{\max _{\Gamma_{c} \times[0, T]}\left|\theta_{c}^{n}\right|}<10^{-6} .
$$

Remark 2 The Picard iteration can be also interpreted as a descent method. It is obvious that a fix-point of the described Picard iteration is a critical or stationary point of the considered minimization problem.

The fix-point iteration i)-iv) with relaxation works well, and the results of the numerical simulations will be demonstrated now.

The Poisson and Helmholtz equations (step ii) and iii) of the Picard iteration) are solved with preconditioned cg-methods or for problems with small dimensions (up to 10000 spatial grid points) with direct solvers for sparse matrices. Typical condition numbers are for example of order $10^{4}$ up to $10^{6}$ for 1000 10000 spatial grid points. Because of the definiteness and the moderate condition numbers of the stiffness matrices the iterative solution methods converge fast also without pre-conditioners.

\section{Results of the numerical solution of the full problem}

\subsection{Optimization of a zone melting configuration}

As a first test problem we consider a zone melting configuration. The used geometrical and material parameters for the crystal $\left(B i_{0.25} S b_{0.75}\right)_{2} T e_{2}$, a composition of bismuth point fifty antimony one point fifty 
tellurium two, are summarized in the Table 1 (see also [6]).

The parameters of Table 1 lead to the Prandtl number $\operatorname{Pr}=8.25 \mathrm{e}-02$ and the Grashof number $G r=1.143 \mathrm{e}+04$. $\left(B i_{0.25} S b_{0.75}\right)_{2} T e_{2}$-crystals are used for small cooling devices. The figure 1 shows the physical domain of the melt zone. The control goal is tracking of a velocity field $\overline{\vec{u}}$, which either is given

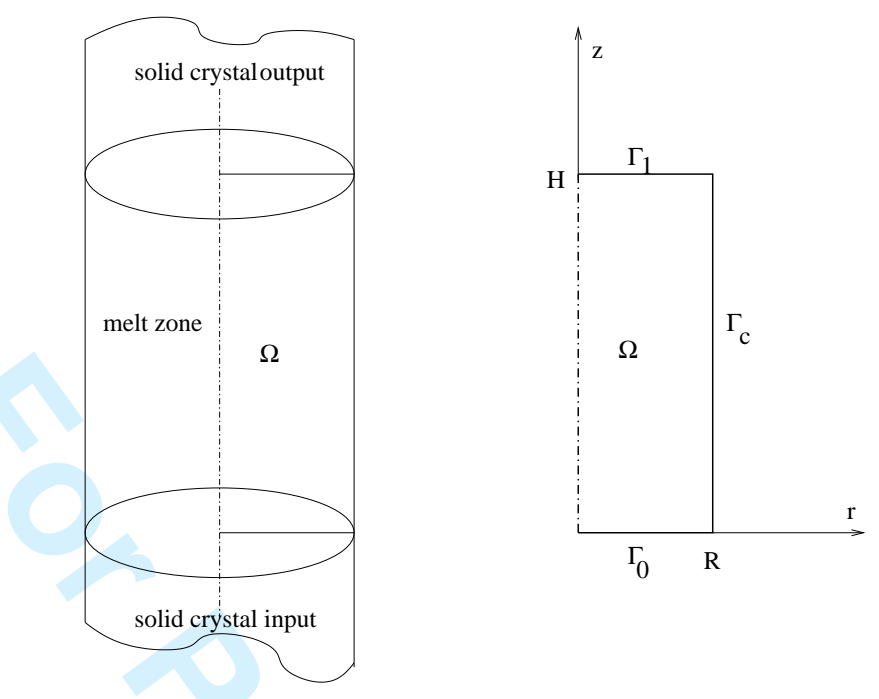

Figure 1. Physical domain for the zone melting growth

by

a) a typical two-dimensional toroidal flow, or by

b) a non moving melt, i.e. $\overline{\vec{u}}=\mathbf{0}$.

The case b) is artificial but serves as a good test case since $\theta_{c}=\theta_{s}=$ const. implies $\vec{u}=\mathbf{0}$, and this velocity field together with $\theta=\theta_{s}$ is a solution of the Boussinesq approximation. Artificial in this context means that $\theta=\theta_{s}$ on $\Omega$ is not a realistic assumption for a crystal melt and the input mixed crystal will never change to a single homogeneous output crystal.

For the velocity we have homogeneous Dirichlet data on the whole boundary. For the temperature we have the boundary conditions

$$
\begin{aligned}
& \theta=\theta_{c}, \text { for } r=R, 0 \leq z \leq H, \varphi \in(0,2 \pi), \\
& \theta=\theta_{s}, \text { for } 0 \leq r \leq R, z=H \\
& \theta=\theta_{s}, \text { for } 0 \leq r \leq R, z=0
\end{aligned}
$$

For $t=0$ we choose the start temperature profile $\theta_{c}=\theta_{c 0}$ on $\Gamma_{c}$ which is given by

$$
\theta_{c 0}(z)=\theta_{s}+4 \frac{z}{H}\left(1-\frac{z}{H}\right) \delta \theta
$$

\begin{tabular}{|l|l|l|}
\hline parameter & symbol & value \\
\hline radius & $R$ & $4.0 \mathrm{e}-03 \mathrm{~m}$ \\
height & $H$ & $1.6 \mathrm{e}-02 \mathrm{~m}$ \\
melting point & $\theta_{s}$ & $613 \mathrm{~K}$ \\
diffusivity & $a$ & $4.4 \mathrm{e}-06 \frac{\mathrm{m}^{2}}{\mathrm{~s}}$ \\
viscosity & $\nu$ & $3.631 \mathrm{e}-07 \frac{\mathrm{m}^{2}}{\mathrm{~s}}$ \\
expansion & $\beta$ & $9.6 \mathrm{e}-05 \mathrm{~K}^{-1}$ \\
\hline
\end{tabular}

Table 1. Parameters of $\left(B i_{0.25} S b_{0.75}\right)_{2} T e_{2}$-melt and of the melt geometry 
with $\theta_{s}=613 K, \delta \theta=25 K$.

The velocity field $\overline{\vec{u}}$, which we want to reach is a typical two-dimensional toroidal flow. We consider a time interval $[0, T]=[0,7.33$ seconds $]$ with $Z=60$ time steps of 0.1222 seconds which means dimensionless time steps of $\tau=1.5 \mathrm{e}-03$. For the spatial discretization we use $20 \times 30$ finite volumes. As results of numerical tests we found the best convergence behavior of the Picard iteration with the damping parameter $\sigma_{r}=0.1$. The regularization was set to $\alpha=0.25$. A further discussion of the interrelation of the regularization and damping parameter is given below.

The Figures 2 and 3 show the results of the optimization problem a), i.e. the resulting control temperature on the boundary-time cylinder and the development of the functional values, where the temperatures are dimensionless defined by $\bar{\theta}=\frac{\theta-\theta_{s}}{\delta}$. In all of the following figures "time" means $\frac{t}{\tau}$ and "height" means $\frac{z}{\Delta z}$.

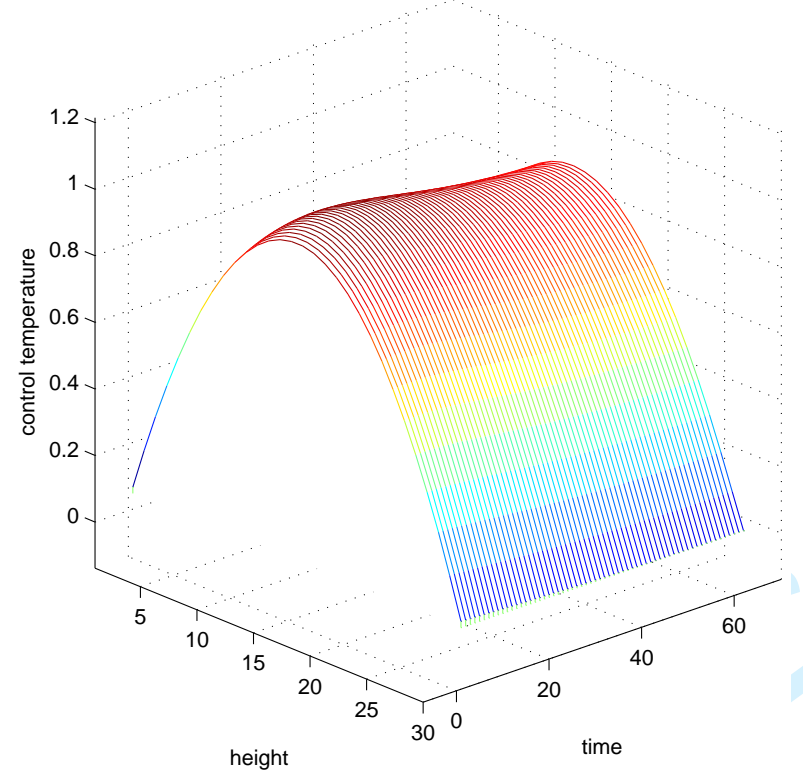

Figure 2. Control temperature, problem a)

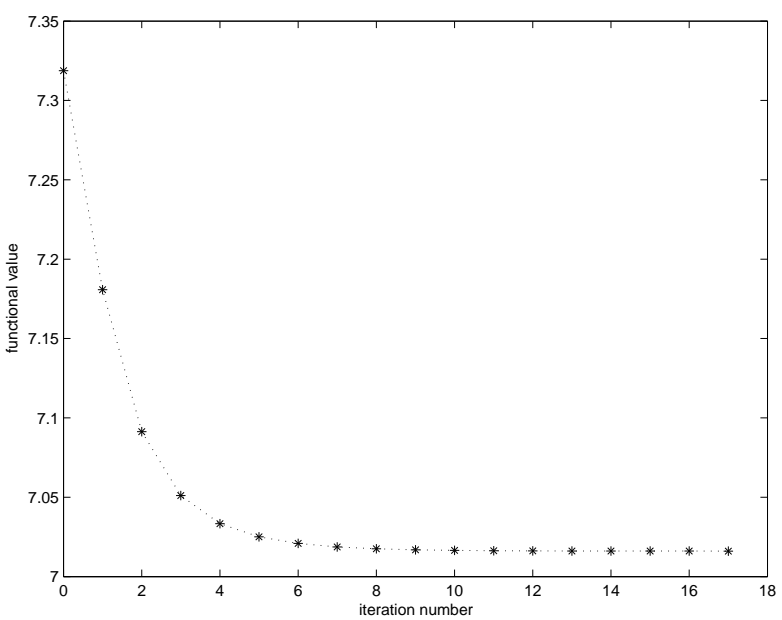

Figure 3. Functional vs. iteration, problem a)

The Figures 4 and 5 show the results of the optimization of problem b). As regularization and damping parameters we used again $\alpha=0.25$ and $\sigma_{r}=0.1$.

\subsection{Optimization of a Czochralski crystal growth process}

As a second test problem we consider a (idealized) Czochralski crystal growth process. The Fig. 6 shows the geometrical configuration of the crucible.

The above discussed model and the optimization system is formulated and implemented in three dimensions. However, because of the huge computational amount of work in three spatial dimensions we first test the optimization procedure for the two-dimensional case $u=0$ (azimuthal component of the velocity) and $\frac{\partial Q}{\partial \varphi}=0$ for all transport quantities $Q(\vec{u}, p, \theta$, etc.). Thus, we consider a two-dimensional spatial domain (see Fig. 6). $R_{c}$ denotes the radius of the solid crystal, $R$ the crucible radius and $H$ is the height of the crystal melt. $\theta_{s}$ denotes the melting point temperature of the crystal material, The geometrical and material parameters are taken as in the Silicon Czochralski growth process described [12] and are summarized in the Table 2.

The material parameters lead to a Prandtl number $\operatorname{Pr}=1.057 \mathrm{e}-02$. The associated Grashof number is given by $1.5 \mathrm{e}+09$ and leads to a strong CFL ( Courant-Friedrichs-Lewy ) condition/restriction for time stepping in our time discretization scheme. Time steps $\tau$ should not be taken larger than 1.0e-05. However, from the practical point of view this requirement is not as restrictive as it seems to be, since one dimensionless time step $\tau=1.0 \mathrm{e}-05$ corresponds to 0.80645 seconds real time. 

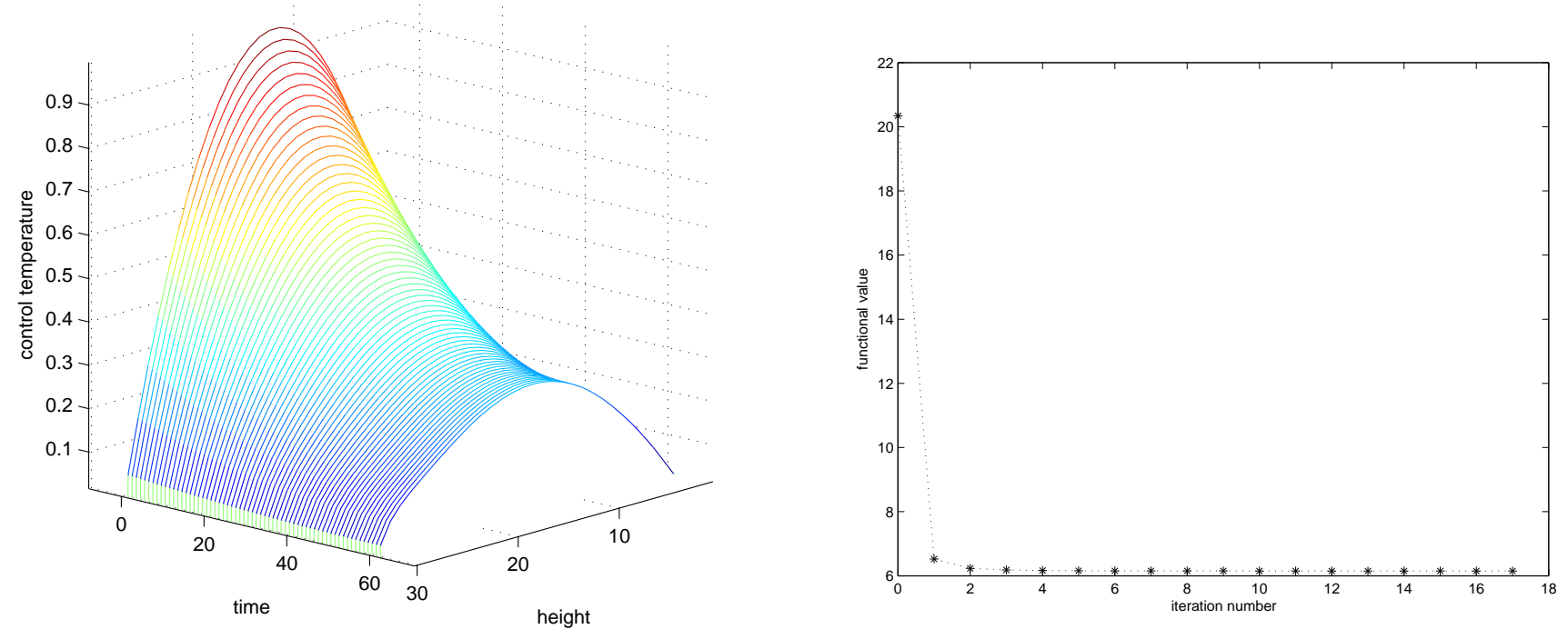

Figure 4. Control temperature problem b)

Figure 5. Functional vs. iteration, problem b)

We investigate Dirichlet boundary control. For the thermal boundary conditions of our Czochralski process we then have

$$
\begin{aligned}
& \theta=\theta_{c} \quad \text { for } r=R, 0 \leq z \leq H, \varphi \in\left[0,2 \pi\left[,\left(\text { control boundary } \Gamma_{c}\right)\right.\right. \\
& \theta=\theta_{s}, \quad \text { for } 0 \leq r \leq R_{c}, z=H,
\end{aligned}
$$
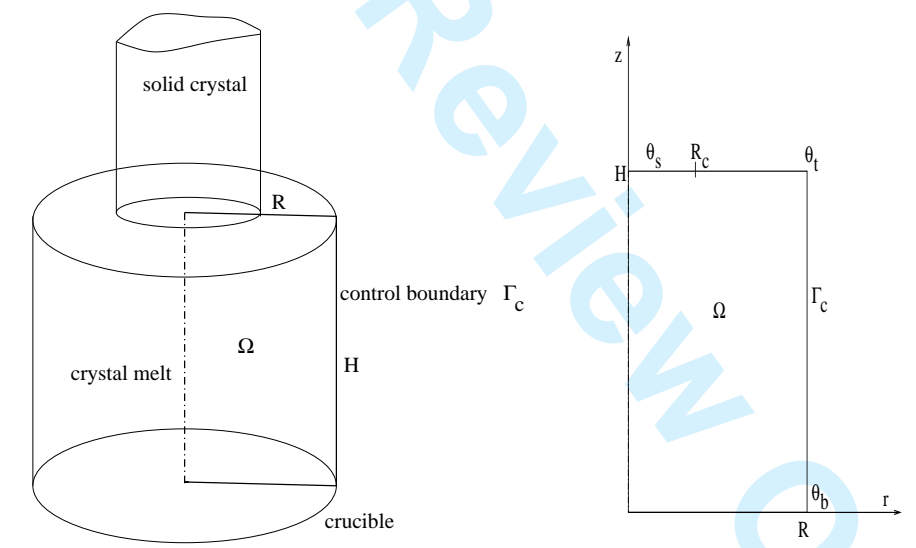

Figure 6. Physical domain for Czochralski growth

\begin{tabular}{|l|l|l|}
\hline parameter & symbol & value \\
\hline crucible radius & $R$ & $1.5 \mathrm{e}-01 \mathrm{~m}$ \\
crystal radius & $R_{c}$ & $7.5 \mathrm{e}-02 \mathrm{~m}$ \\
height of the melt & $H$ & $4.0 \mathrm{e}-01 \mathrm{~m}$ \\
melting point temperature & $\theta_{s}$ & $1683 \mathrm{~K}$ \\
thermal diffusivity & $a$ & $2.64 \mathrm{e}-05 \frac{\mathrm{m}^{2}}{\mathrm{~s}}$ \\
kinematic viscosity & $\nu$ & $2.79 \mathrm{e}-07 \frac{\mathrm{m}^{2}}{\mathrm{~s}}$ \\
thermal expansion coefficient & $\beta$ & $1.41 \mathrm{e}-04 \mathrm{~K}^{-1}$ \\
\hline
\end{tabular}

Table 2. Parameters of Silicon and of the melt geometry 

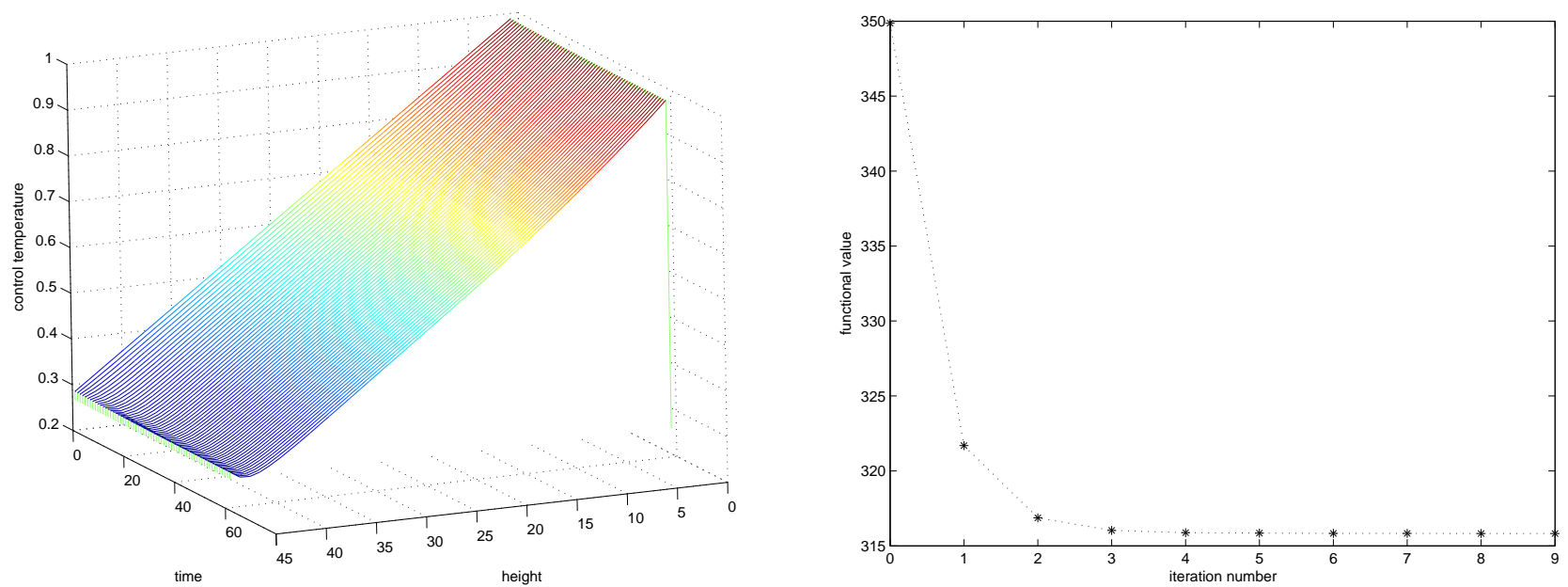

Figure 7. Result of the optimization of the Czochralski process, second test problem

$$
\begin{aligned}
& \theta=\theta_{s}+\frac{r-R_{c}}{R-R_{c}}\left(\theta_{t}-\theta_{s}\right), \text { for } R_{c} \leq r \leq R, z=H, \\
& \theta=\theta_{t}, \quad \text { for } 0 \leq r \leq R, z=0 .
\end{aligned}
$$

For $t=0$ we choose the start temperature profile $\theta_{c}=\theta_{c 0}$ on $\Gamma_{c}$ which is given by

$$
\theta_{c 0}(z)=\theta_{b}+\frac{z}{H}\left(\theta_{t}-\theta_{b}\right)
$$

with $\theta_{t}=1690 K, \theta_{b}=1708 K\left(\theta_{s}\right.$ s. Table 2$)$.

As a desired velocity field we use $\overline{\vec{u}}=\mathbf{0}$. The spatial grid has the dimension $20 \times 45$.

The Fig. 7, left shows the temperature distribution $\theta_{c}$ on $\Gamma_{c T}$ for a time horizon containing 90 time steps $(\approx 68$ seconds). In this computations $\alpha=0.25$ is taken as regularization parameter, and the relaxation parameter is chosen $\sigma_{r}=0.75$. Fig. 8 compares the optimal control temperature profiles at time $t=0$ to those at $t=T$ (endpoint of the considered time interval) for different regularization parameters $\alpha$. One observes that the profiles at time $t=T$ become slightly nonlinear compared to the linear profile at $t=0$. The temperatures $\bar{\theta}$ in the Fig. 7 are dimensionless i.e.

$$
\bar{\theta}=\frac{\theta-\theta_{b}}{\theta_{t}-\theta_{b}} .
$$

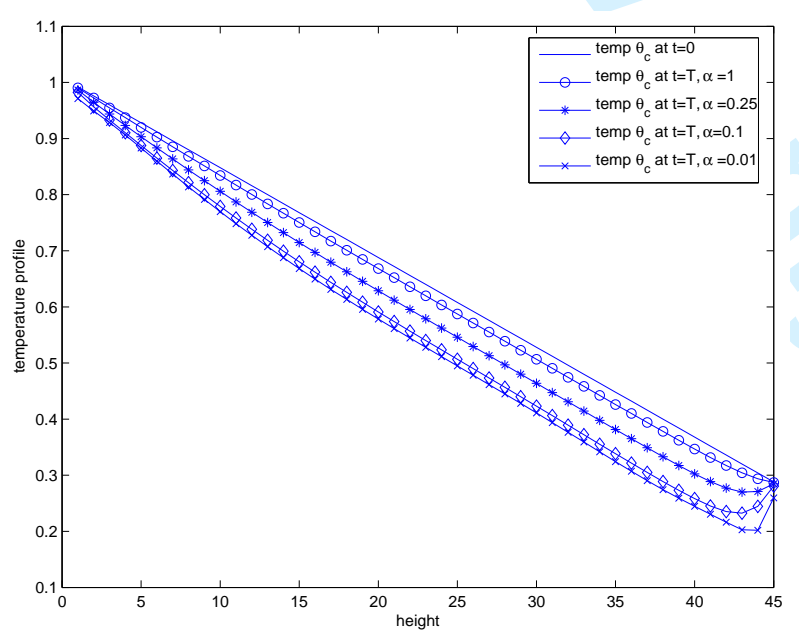

Figure 8. Result of the optimization of the Czochralski process, second test problem 
Finally, we briefly report on the influence of the regularization parameter $\alpha$ on the control process. First we observe, that a reduction of $\alpha$ correlates to a decrease of the damping parameter $\sigma_{r}$ in the Picard iteration. In the present example we use $\sigma_{r}=0.9$ for $\alpha=1.0, \sigma_{r}=0.75$ for $\alpha=0.25, \sigma_{r}=0.4$ for $\alpha=0.1$, and $\sigma_{r}=0.1$ for $\alpha=0.01$.

However, it turns out that the quality of the controls does not change significantly with decreasing $\alpha$, at least in the investigated parameter range $\alpha \in] 0.01,1[$, compare Fig. 7, right, where the final control temperature profiles at $t=T$ are presented. With the same figure we can report an increase of the gradient

$$
\left.\frac{\partial \theta_{c}}{\partial z}\right|_{\{z=H, t=T\}},
$$

at height $z=H$ and time $t=T$ with decreasing $\alpha$, while for $\left.\frac{z}{\Delta z} \in\right] 0,35$ [ the temperature profiles remain nearly linear. These results to a certain extent justify the Ansatz of boundary temperature control for Czochralski growth presented in [13], where the slope of a linear boundary temperature profile serves as an optimization variable. However, the Fig. 7, right, where the development of the functional values during the optimization process for $\alpha=0.25$ and $\sigma_{r}=0.4$ is reported, shows in the present case $(\overrightarrow{\vec{u}}=\overrightarrow{0})$ a considerable decrease of the functional value.

For toroidal desired velocity fields (case a)) we observe that the decrease of the functional value during the optimization process is very small or even negligible. This also is in accordance with the numerical findings of [13] for boundary temperature control.

\section{Conclusion}

With the Lagrange parameter technique it's possible to derive an optimization system for a given functional whose solution gives an optimal control. The numerical examples of the fully time-depend $2.5 \mathrm{~d}$ optimization system show the possibility of the practical optimization of a thermal coupled flow problem in the crystal growth field.

The results make sense physically and show the possibility of boundary control in the cases of the zone melting technique and the Czochralski method. For the Czochralski growth technique the results show that also small differences between the temperature profiles $\theta_{c}(0)$ and $\theta_{c}(T)$ can lead to certain optimization effects related to the functional values. Based on the results with the proposed strategies it is now possible to do a fully $3 \mathrm{~d}$ optimization.

Because of the lack of other results for a comparison or validation we are in discussion with physicists and engineers to set up crystal growth experiments using our results.

However it is necessary to continue numerical experiments to investigate the utility of the optimization during a pure boundary control as a successful technology. There are some experiences with other optimization problems which show the efficiency of volume control, if there is a possibility of the goaloriented generation of volume forces (for example by a magnetic field). Experimental investigations of such phenomena are presented in [14].

The presented optimization method is applicable to other coupled transport problems for example the coupling of solutal and natural convection.

The dicretized optimization system consisting of a large coupled nonlinear equation system could be solved with a Picard iteration. Especially in the case of the consideration of larger time intervals and finer spatial grids as used in the examples it is necessary in a future work to investigate other steepest descend solution techniques with step size control. On the other hand the problem of constraints for the state and/or the control variables should be considered in the future by a rigorous mathematical analysis and by the construction of appropriate solution methods (non smooth newton, inner point methods). Regarding the considered application it is important to add phenomens like the radiation and volume forces created by magnetic field strength to the Boussinesq equation system. 


\section{Acknowledgments}

This work is part of a cooperation with Prof. Mcheal Hinze from the Universität Hamburg. The topic of the work has been partially supported by the Mathematisches Forschungszentrum Matheon granted by the Deutsche Forschungsgemeinschaft.

We have to thank Dr. Klaus Böttcher and Prof. Peter Rudolph from the Leibniz Institut für Kristallzüchtung Berlin for many helpful discussions about the physics of crystal growth.

Finally, we thank the anonymous referees for the valuable comments which helped to improve the presentation of the material.

\section{References}

[1] Gajewski, H., Zur iterativen Lösung der zweidimensionalen Boussinesq-Gleichungen. ZAMM, 55 (1975)

[2] Ladyzhenskaya, O., The Mathematical Theory of Viscous Incompressible Flows. Gordon \& Breach, 1969

[3] Temam, R., Navier-Stokes Equations. North-Holland, 1979

[4] Bärwolff, G. and Hinze, M., Optimization of semiconductor melts. ZAMM, 86 (2006) 6

[5] Bärwolff, G. and Hinze, M., Analysis of an optimization problem with the Boussinesq equation. to appear in $Z A M M$

[6] Bärwolff, G., König, F. and Seifert, G., Thermal buoyancy convection in vertical zone melting configurations. ZAMM, 77 (1997) 10

[7] Tröltzsch, F., Optimale Steuerung partieller Differentialgleichungen. Vieweg, 2005

[8] Hinze, M., Optimal and instantaneous control of the in-stationary Navier-Stokes equations, habilitation thesis Berlin. Available online at ww. math.tu-dresden.de/ ${ }^{\sim}$ hinze (accessed August 2000)

[9] Hinze, M. and Kunisch, K., Second order methods for optimal control of time-dependent fluid flow. SIAM J. Control Optim., 40 (2001).

[10] Kurz, M.R.H. and Müller, G., Control of thermal conditions during crystal growth by inverse modeling. Journal of Crystal Growth, 208 (2000)

[11] Constantin, P. and Foias, C., Navier-Stokes Equations. The University of Chicago Press, 1988

[12] Böttcher, K., Collected solid crystal and crystal melt material parameters. Berlin, 2003. Available online at http: //www.ikz-berlin.de/ ${ }^{\mathrm{kb}}$

[13] Gunzburger, M., Ozugurlu, E., Turner, J. and Zhang, H., Controlling transport phenomena in the Czochralski crystal growth process. Journal of Crystal Growth 234 (2002)

[14] Eckert, Gerbeth, G., Velocity measurements in liquid sodium by means of ultrasound Doppler velocimetry. Experiments in Fluids, $32(2002)$ 


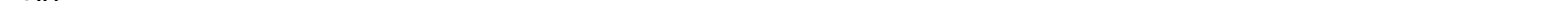




\section{International Journal of Computer MathematicsPage 14 of 21}

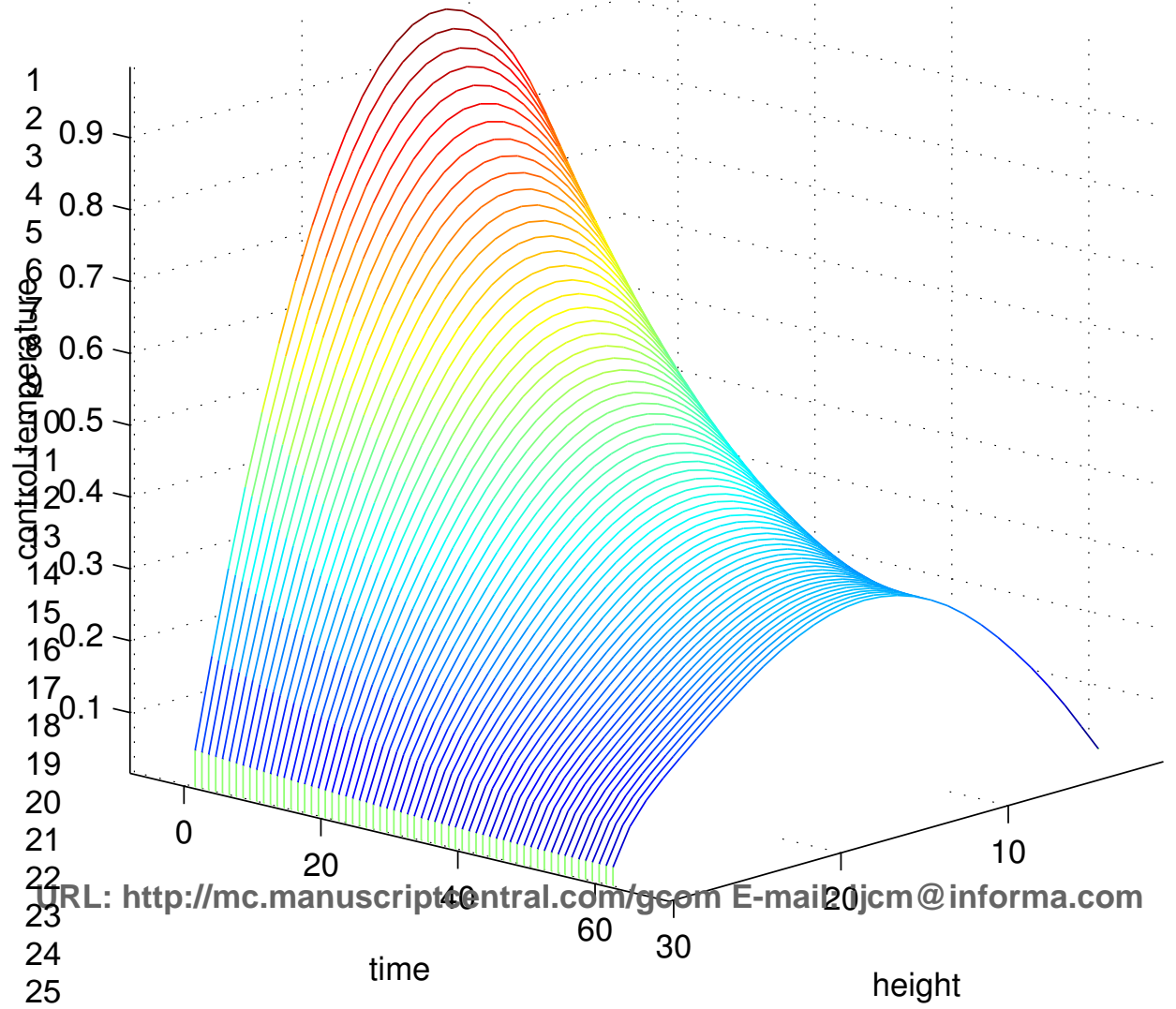




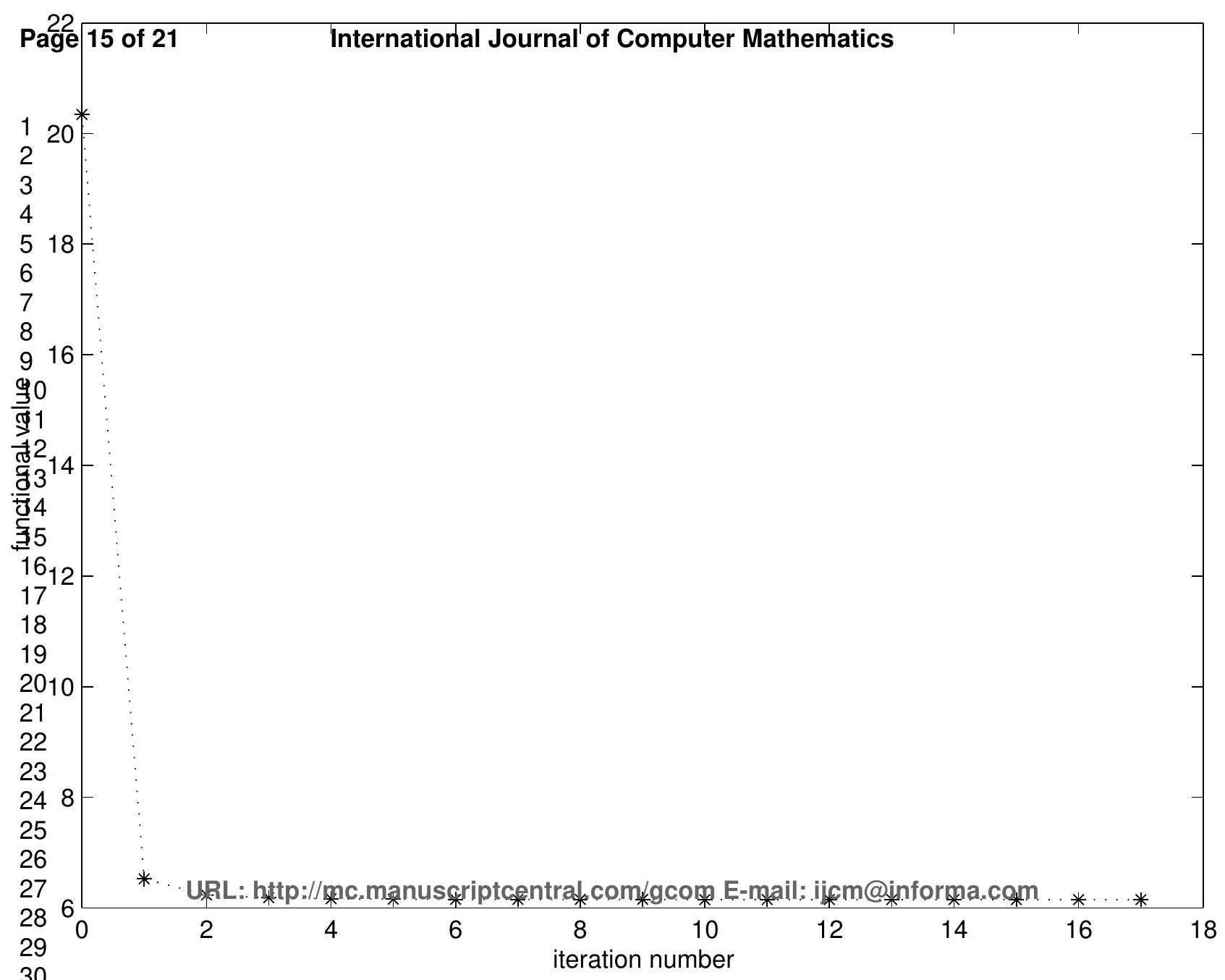




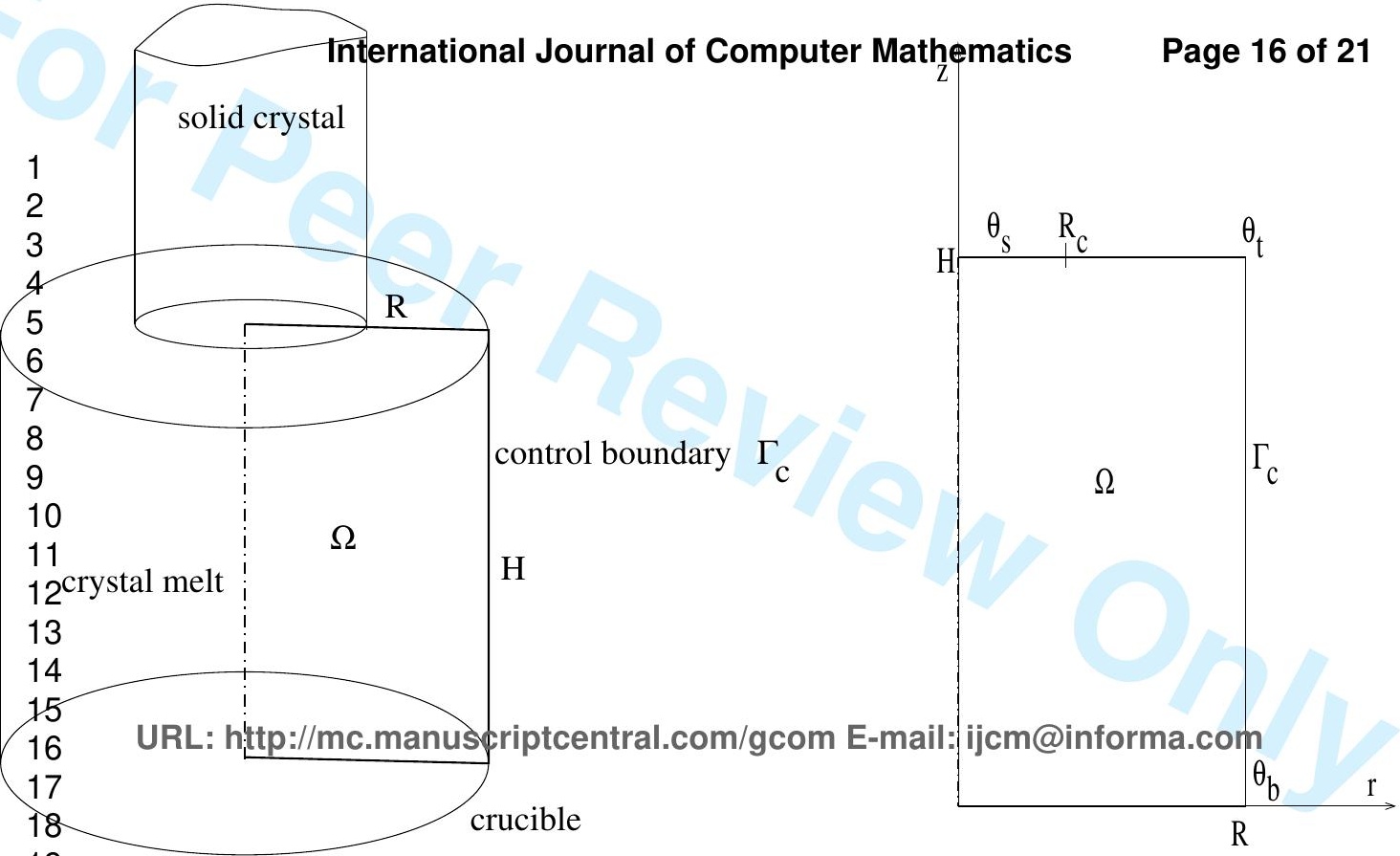








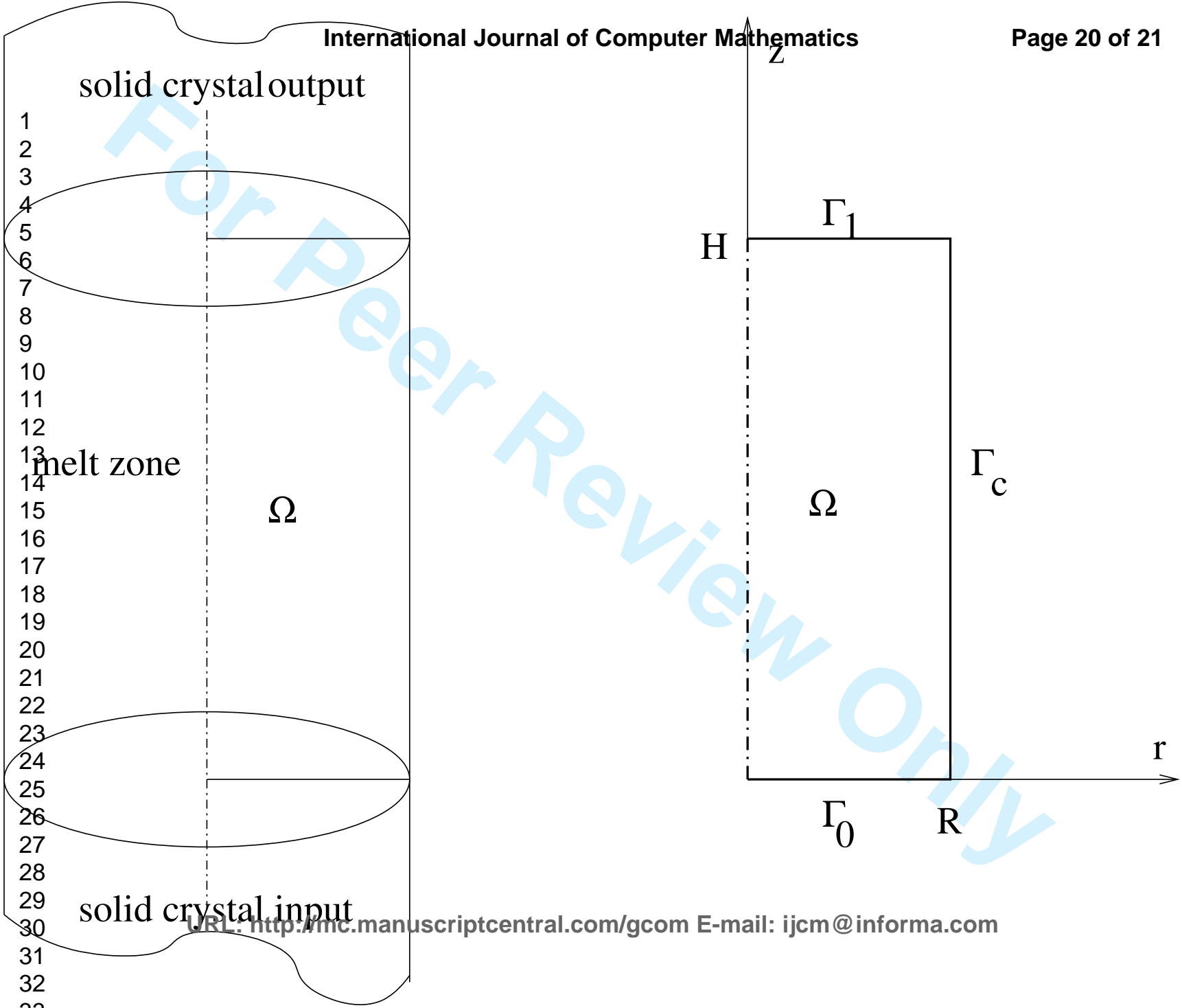
